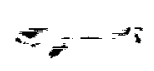

NASA Technical Memorandum 109017

ICASE Report No. 93-49

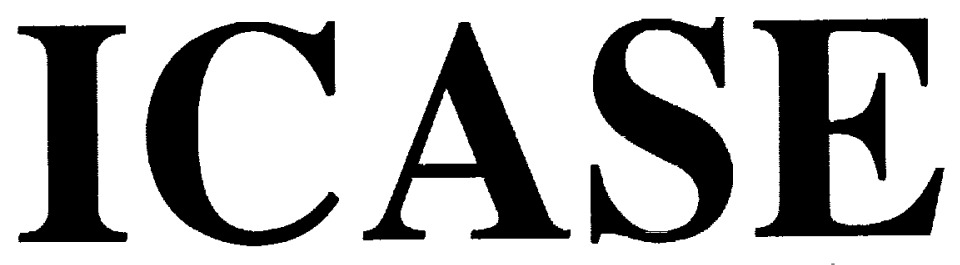

$1 N-34$

191148

\title{
BROKEN SYMMETRY IN IDEAL MAGNETOHYDRODYNAMIC TURBULENCE
}

John V. Shebalin

(NASA-TM-109017) BROKEN SYMMETRY

N94-15964

IN IDEAL MAGNETCHYDROOYNAMIC

TURBULENCE (ICASE) $17 \mathrm{P}$

Unclas

65

$\mathbb{E}_{1} / 34 \quad 0191148$

August 1993

Institute for Computer Applications in Science and Engineering

NASA Langley Research Center

Hampton, Virginia 23681-0001

Operated by the Universities Space Research Association

NASA

National Aeronautics and

Space Adminlstration

Langley Research Center

Hampton, VIrglnla 23681-0001 
r. . . 


\section{ICASE Fluid Mechanics}

Due to increasing research being conducted at ICASE in the field of fluid mechanics, future ICASE reports in this area of research will be printed with a green cover. Applied and numerical mathematics reports will have the familiar blue cover, while computer science reports will have yellow covers. In all other aspects the reports will remain the same; in particular, they will continue to be submitted to the appropriate journals or conferences for formal publication. 


\title{
Broken Symmetry in Ideal Magnetohydrodynamic Turbulence
}

\author{
John V. Shebalin* \\ National Aeronautics and Space Administration \\ Langley Research Center \\ Hampton, Virginia 23681, USA
}

A numerical study of the long-time evolution of a number of cases of inviscid, isotropic, incompressible, three-dimensional fluid and magneto-fluid turbulence has been completed. The results confirm that ideal magnetohydrodynamic turbulence is non-ergodic if there is no external magnetic field present. This is due essentially to a canonical symmetry being broken in an arbitrary dynamical representation. The broken symmetry manifests itself as a coherent structure, i.e., a non-zero time-averaged part of the turbulent magnetic field. The coherent structure is observed, in one case, to contain about eighteen percent of the total energy.

\footnotetext{
"Research supported by the National Aeronautics and Space Administration. This work was performed while the author was in residence as a Visiting Scientist at the Institute for Computer Applications in Science and Engineering (ICASE), NASA Langley Research Center, Hampton, VA 23681.
} 


\section{Introduction}

Several years ago, a numerical study of isotropic, inviscid, incompressible, two-dimensional (2D) turbulence indicated that ideal magnetohydrodynamic (MHD) turbulence was non-ergodic [1]. A Fourier spectral method was used, and the non-ergodicity manifested itself in the appearance of significant, non-zero timeaverages for the Fourier coefficients of the turbulent magnetic field. These results contrasted with the predictions of canonical ensemble theory [2] and indicated the presence of a dynamically broken symmetry.

Here, this work is extended in two ways. First, the dynamics become fully three-dimensional (3D), and second, a more accurate (third-order) time-integration scheme is used (a comparison is made with the second-order scheme used previously). The results are qualitatively the same as previously seen, although a stronger effect is observed. Also, the source of the broken symmetry is more explicitly identified.

\section{Basic Equations}

The equations which describe ideal, incompressible 3D fluid and magneto-fluid dynamics are

$$
\begin{aligned}
\partial_{t} \mathbf{B} & =\nabla \times(\mathbf{u} \times \mathbf{B}) \\
\partial_{t} \boldsymbol{\omega} & =\nabla \times(\mathbf{u} \times \boldsymbol{\omega}+\mathbf{j} \times \mathbf{B})
\end{aligned}
$$

Here, the fluid velocity is $\mathbf{u}$, the vorticity is $\boldsymbol{\omega}=\nabla \times \mathbf{u}$, the magnetic field is $\mathbf{B}=\mathbf{b}+\mathbf{B}_{\circ}$ (where $\mathbf{b}$ is the turbulent part and $\mathbf{B}_{o}$ is a constant, externally imposed part), and the current is $\mathbf{j}=\nabla \times \mathbf{b}$. Also, $\nabla \cdot \mathbf{u}=\nabla \cdot \mathbf{B}=0$

If $\mathbf{B} \equiv 0$ for all time, then (1) is identically satisfied and (2) assumes the vorticity form of the incompressible Euler equation. If $\mathbf{B}_{0} \equiv 0$ while $\mathbf{b} \neq 0$, then (1) and (2) are unchanged, i.e., symmetric, under the substitution $\mathbf{b} \rightarrow-\mathbf{b}$. If, however, $\mathbf{B}_{\circ} \neq 0$ also, then the equations are no longer symmetric under $\mathbf{b} \rightarrow-\mathbf{b}$.

Note that these equations are inviscid. It is well known that solutions to these equations are quite different from those for dissipative equations, even if the viscosity and resistivity become very small, so long as they are not identically zero. However, it is possible to determine a priori statistical solutions in the inviscid case (as will be discussed presently), while it has not proven possible to do this in the dissipative case. 
This provides for a very practical use of the results presented herein, that is, as a test of the correctness of any computer code which attempts to simulate incompressible, homogeneous, dissipative turbulence. In any such code, the viscosity (and resistivity) can be set identically to zero; the statistical properties of a numerical solution must then be described by the inviscid theory described here. There are, however, some refinements which must be incorporated into existing theory; these refinements, the existence of broken symmetry and non-ergodicity in ideal (3D) MHD turbulence, are the subject of this paper.

\section{Canonical Ensembles}

The theory of canonical or 'absolute equilibrium' ensembles, as it applies to homogeneous turbulence, has been discussed extensively before $[1,3]$ (and the many references therein). In brief, the physical variables $\mathbf{u}$, $\mathbf{b}, \boldsymbol{\omega}$, and $\mathbf{j}$ are expanded in truncated Fourier series, for example:

$$
\mathbf{b}(\mathbf{x}, t)=\frac{1}{N^{3 / 2}} \sum_{k_{m i n}}^{k_{\max }} \mathbf{b}(\mathbf{k}, t) e^{i \mathbf{k} \cdot \mathbf{x}}
$$

Here, the sum (and each similar sum appearing henceforth) is over all $\mathbf{k}$ such that $k_{\min } \leq|\mathbf{k}| \leq k_{\max }<N / 2$, where $N$ is the number of points in each of the three spatial dimensions. Since the various fields, such as $\mathbf{b}(\mathbf{x})$, are real, their coefficients satisfy $\mathbf{b}(\mathbf{k})=\mathbf{b}^{*}(-\mathbf{k})$, where ${ }^{\text {(*) }}$ denotes complex conjugation (here and henceforth, explicit time-dependence will be dropped for brevity). Also, $\mathbf{u}(\mathbf{k})=i k^{-2} \mathbf{k} \times \boldsymbol{\omega}(\mathbf{k})$ and $\mathbf{j}(\mathbf{k})=i \mathbf{k} \times \mathbf{b}(\mathbf{k})$, and $\nabla \cdot \mathbf{b}(\mathbf{x})=0 \rightarrow i \mathbf{k} \cdot \mathbf{b}(\mathbf{k})=0$, etc.

The independent real and imaginary parts of the coefficients $\mathbf{u}(\mathbf{k})$ and $\mathbf{b}(\mathbf{k})$ can be used to label the axes of a multidimensional phase space. The corresponding dynamical system is described by a single point in the phase space, which moves about as the system evolves in time. The probability that the system point is in any part of phase space can be described by a canonical distribution function which depends only on a small set of conserved quantities, the integral invariants of the dynamical system. Once the joint probability distribution has been found, then the equilibrium energy spectra (kinetic and magnetic) are determined, even though $\mathbf{u}(\mathbf{k})$ and $\mathbf{b}(\mathbf{k})$ are random variables.

In the case of isotropic, incompressible 3D Euler turbulence $(\mathbf{B} \equiv 0)$, the integral invariants are the energy $E$ and the kinetic helicity $H_{k}$. For ideal, isotropic, incompressible 3D MHD turbulence with $\mathbf{B}_{o} \equiv 0$, the integral invariants are the energy $E$, the cross helicity $H_{c}$, and the magnetic helicity $H_{m}$; if $\mathbf{B}_{o} \neq 0$, then 
$H_{m}$ is no longer conserved, although $E$ and $H_{c}$ are still integral invariants. These three different situations will now be presented in more detail.

\subsection{Euler Turbulence}

The integral invariants for incompressible $3 \mathrm{D}$ Euler turbulence $[4,5]$ are

$$
E=\frac{1}{2 N^{3}} \sum_{k_{\min }}^{k_{\max }}|\mathbf{u}(\mathbf{k})|^{2} \text { and } H_{k}=\frac{1}{2 N^{3}} \sum_{k_{\min }}^{k_{\max }} \mathbf{u}(\mathbf{k}) \cdot \boldsymbol{\omega}^{\prime \prime}(\mathbf{k}),
$$

while the distribution function is

$$
D=C \exp \left(-\alpha E-\beta H_{k}\right) \quad \text { where } \quad C=\prod_{k_{\min }}^{k_{\max }}\left(\frac{\alpha^{2}-\beta^{2} k^{2}}{\pi^{2} k^{4} N^{6}}\right)^{\frac{3}{2}} .
$$

The product above (and each product appearing henceforth) is taken over all $\mathbf{k}$ such that $k_{\min } \leq|\mathbf{k}| \leq k_{\max }$, but only over independent values of $\mathbf{k}$, i.e., if $\mathbf{k}$ is used, then $-\mathbf{k}$ is not. Using this distribution function, an expectation value can be defined as

$$
\langle Q\rangle \equiv \int Q D \prod_{k_{\min }}^{k_{\max }} d^{3} \mathbf{u}(\mathbf{k})
$$

with which expectation values of the moments of the Fourier coefficients can be found:

$$
\begin{gathered}
\left\langle\mathbf{u}_{R}(\mathbf{k})\right\rangle=\left\langle\mathbf{u}_{I}(\mathbf{k})\right\rangle=0, \quad\left\langle\left|\mathbf{u}_{R}(\mathbf{k})\right|^{2}\right\rangle=\left\langle\left|\mathbf{u}_{I}(\mathbf{k})\right|^{2}\right\rangle=\frac{3 N^{3} \alpha}{2\left(\alpha^{2}-\beta^{2} k^{2}\right)}, \\
\text { and } \quad\left\langle\mathbf{u}_{R}(\mathbf{k}) \cdot \omega_{R}(\mathbf{k})\right\rangle=\left\langle\mathbf{u}_{I}(\mathbf{k}) \cdot \omega_{I}(\mathbf{k})\right\rangle=\frac{-3 N^{3} \beta k^{2}}{2\left(\alpha^{2}-\beta^{2} k^{2}\right)}
\end{gathered}
$$

where the subscripts $R$ and $I$ denote real and imaginary parts, respectively, of the complex coefficients, and where all the components of $\mathbf{u}(\mathbf{k})$ for a given $\mathbf{k}$ have equal expectation values. A straightforward algebraic manipulation of these expectation values yields

$$
\alpha=\frac{Z\langle\Omega\rangle}{\langle E\rangle\langle\Omega\rangle-\left\langle H_{k}\right\rangle^{2}} \quad \text { and } \quad \beta=-\frac{\left\langle H_{k}\right\rangle}{\langle\Omega\rangle} \alpha
$$

where

$$
Z=\frac{1}{2 N^{3}} \sum_{k_{m i n}}^{k_{\max }} 1 \text { and } \Omega=\frac{1}{2 N^{3}} \sum_{k_{m i n}}^{k_{\max }} k^{2}|\mathbf{u}(\mathbf{k})|^{2} .
$$

Note that the expected value of the mean squared vorticity (the enstrophy $\Omega$ ) is determined once the expectation values (7) of the $|\mathbf{u}(\mathbf{k})|^{2}$ are known. 
Although the enstrophy $\Omega$ is not a conserved quantity, it does have an expectation value, so that the parameters $\alpha$ and $\beta$ are functions of only one unknown quantity, $\langle\Omega\rangle$. The quantity $Z$ is the ratio of the number of independent values of $\mathbf{k}$ to $N^{3}$, the total number of points in the spatial grid.

\subsection{Ideal MHD Turbulence}

The integral invariants of ideal, incompressible 3D MHD turbulence with $\mathbf{B}_{o}=0[6]$ are

$$
\begin{gathered}
E=\frac{1}{2 N^{3}} \sum_{k_{m i n}}^{k_{\max }}\left[|\mathbf{u}(\mathbf{k})|^{2}+|\mathbf{b}(\mathbf{k})|^{2}\right], \quad H_{c}=\frac{1}{2 N^{3}} \sum_{k_{\min }}^{k_{\max }} \mathbf{u}(\mathbf{k}) \cdot \mathbf{b}^{*}(\mathbf{k}), \\
\text { and } \quad H_{m}=\frac{1}{2 N^{3}} \sum_{k_{\min }}^{k_{\max }} k^{-2} \mathbf{j}(\mathbf{k}) \cdot \mathbf{b}^{*}(\mathbf{k})
\end{gathered}
$$

while the distribution function is

$$
D=C \exp \left(-\alpha E-\beta H_{c}-\gamma H_{m}\right), \quad \text { where } \quad C=\prod_{k_{m, n}}^{k_{m a x}}\left[\frac{\left(\alpha^{2}-\beta^{2} / 4\right)^{2}-\alpha^{2} \gamma^{2} / k^{2}}{\pi^{4} N^{12}}\right]^{\frac{3}{2}}
$$

(Please note that the formulas appearing in this subsection correct those which appeared previously [1].)

Using this distribution function, expectation values (where (6) now includes integration over the independent components of the $\mathbf{b}(\mathbf{k}))$ for moments of the Fourier coefficients can be found:

$$
\begin{gathered}
\left\langle\mathbf{u}_{R}(\mathbf{k})\right\rangle=\left\langle\mathbf{u}_{I}(\mathbf{k})\right\rangle=0, \quad\left\langle\left|\mathbf{u}_{R}(\mathbf{k})\right|^{2}\right\rangle=\left\langle\left|\mathbf{u}_{I}(\mathbf{k})\right|^{2}\right\rangle=\frac{3}{2} N^{3}\left[\frac{\alpha\left(\alpha^{2}-\beta^{2} / 4-\gamma^{2} / k^{2}\right)}{\left(\alpha^{2}-\beta^{2} / 4\right)^{2}-\alpha^{2} \gamma^{2} / k^{2}}\right] \\
\left\langle\mathbf{b}_{R}(\mathbf{k})\right\rangle=\left\langle\mathbf{b}_{I}(\mathbf{k})\right\rangle=0, \quad\left\langle\left|\mathbf{b}_{R}(\mathbf{k})\right|^{2}\right\rangle=\left\langle\left|\mathbf{b}_{I}(\mathbf{k})\right|^{2}\right\rangle=\frac{3}{2} N^{3}\left[\frac{\alpha\left(\alpha^{2}-\beta^{2} / 4\right)}{\left(\alpha^{2}-\beta^{2} / 4\right)^{2}-\alpha^{2} \gamma^{2} / k^{2}}\right] \\
\left\langle\mathbf{u}_{R}(\mathbf{k}) \cdot \mathbf{b}_{R}^{*}(\mathbf{k})\right\rangle=\left\langle\mathbf{u}_{I}(\mathbf{k}) \cdot \mathbf{b}_{I}^{*}(\mathbf{k})\right\rangle=\frac{3}{4} N^{3}\left[\frac{-\beta\left(\alpha^{2}-\beta^{2} / 4\right)}{\left(\alpha^{2}-\beta^{2} / 4\right)^{2}-\alpha^{2} \gamma^{2} / k^{2}}\right] \\
\left\langle\mathbf{j}_{R}(\mathbf{k}) \cdot \mathbf{b}_{R}^{*}(\mathbf{k})\right\rangle=\left\langle\mathbf{j}_{I}(\mathbf{k}) \cdot \mathbf{b}_{I}^{*}(\mathbf{k})\right\rangle=\frac{3}{2} N^{3}\left[\frac{-\alpha^{2} \gamma}{\left(\alpha^{2}-\beta^{2} / 4\right)^{2}-\alpha^{2} \gamma^{2} / k^{2}}\right]
\end{gathered}
$$

where all the components of $\mathbf{u}(\mathbf{k})$ for a given $\mathbf{k}$ have equal expectation values, and where all the components of $\mathbf{b}(\mathbf{k})$ for a given $\mathbf{k}$ also have equal expectation values. Note that the energy spectra in the MHD case (11) peak at low $k=|\mathbf{k}|$, while in the Euler case (7), it peaks at high $k$. 
A straightforward algebraic manipulation of these expectation values yields

$$
\begin{array}{cc}
\alpha=\frac{Z R(R+1)\langle E\rangle}{2\left[R\langle E\rangle^{2}-(R+1)^{2}\left\langle H_{c}\right\rangle^{2}\right]}, \quad \beta=-2 \frac{(R+1)\left\langle H_{c}\right\rangle}{R\langle E\rangle} \alpha, \\
\gamma=-\frac{(R-1)\langle E\rangle}{(R+1)\left\langle H_{m}\right\rangle} \alpha, \quad \text { where } \quad R=\frac{\left\langle E_{m}\right\rangle}{\left\langle E_{k}\right\rangle} .
\end{array}
$$

Thus $\alpha, \beta$, and $\gamma$ are functions of only one unknown quantity, $R$, which is the ratio of the expectation values of the magnetic part of the total energy to kinetic part of the total energy.

The integral invariants of ideal, incompressible 3D MHD turbulence with $\mathbf{B}_{\circ} \neq 0$ are only $E$ and $H_{c}$, as given above, while $H_{m}$ is no longer conserved. In this case, $R=1$ and $\gamma=0$.

\subsection{Distribution Parameters}

The parameters $\alpha, \beta$, and $\gamma$, which appear in the canonical distribution functions, must be given specific values in order that definite predictions can be made. This can be done in a number of ways. First, it can be assumed that the integral invariants of the various cases have fixed values (although they actually fluctuate slightly in the time evolution of a canonical system). The distribution parameters then vary only with respect to a single quantity: $\langle\Omega\rangle$ for 3D Euler turbulence and $R$ for ideal 3D MHD turbulence (except for the case where $\mathbf{B}_{o} \neq 0$, where $R \equiv 1$ ). The equilibrium entropy [1], $S=S_{o}-\log C$, where $C$ is the normalizing coefficient of the distribution function, is thus also a function of a single quantity. Since $S$ is a minimum with respect to the distribution parameters [7], then this fact can be used to uniquely determine $\langle\Omega\rangle$ or $R$, as the case may be. Note that this can be done prior to any numerical simulation of the time evolution of an isotropic, inviscid turbulent system.

A second method is to run a numerical simulation and actually determine the time-averaged values $\bar{Q}$ of all the quantities $Q$ which are required, so that $\bar{Q}$ can be substituted for $\langle Q\rangle$. In particular, $\bar{\Omega} \rightarrow\langle\Omega\rangle$ or $\bar{R} \rightarrow R$. A third, and more practical method, is to use the time-averaged values of the necessary integral invariants, but determine $\langle\Omega\rangle$ or $R$ through a least-squares procedure. This third method will turn out to be the most useful.

Once the canonical predictions of the moments of $\mathbf{u}(\mathbf{k})$ and $\mathbf{b}(\mathbf{k})$ are available, and numerical simulations have produced corresponding time-averages, then comparisons can be made. This is essentially a test of the 
ergodicity of the various dynamical systems, i.e., the equivalence of ensemble and time averages. Numerical results, including these time averages, are presented next, followed by a discussion addressing any anomalies which are observed.

\section{Numerical Results}

As in the previous work [1], a Fourier spectral method [9] with shifted-grid dealiasing [10] was used to solve the dynamical equations numerically. In the previous effort, however, the time-integration method was a second-order Runge-Kutta scheme (RK2), while the method primarily employed in this work was a third-order scheme, consisting of an Adams-Bashforth predictor coupled with an Adams-Moulton corrector (AB3) [8]. The advantages of AB3 over RK2 are higher accuracy and higher speed, although slightly more storage was required in computer memory. In order to better relate the $3 \mathrm{D}$ results obtained here to the $2 \mathrm{D}$ ones obtained previously [1], two 3D runs which had used AB3 were partially re-run using RK2, with initial conditions remaining the same.

The numerical simulations performed in the course of this work are presented in Table 1. All runs were done on a Cray YMP, using a $16^{3}$ grid with $k_{\max }^{2}=56$ and $\Delta t=0.001$. Each run is designated by three characters: the first is either $\mathrm{E}$ (for Euler) or $\mathrm{M}$ (for MHD), the second is a number representing a different set of initial conditions, and the last denotes the time-integration method used, A (for AB3) or R (for RK2). (The 'initial conditions' include the values of $B_{o}$ and $k_{\min }$, as well as $\left\{\omega(\mathbf{k}), \mathbf{b}(\mathbf{k}) ; k_{\min } \leq|\mathbf{k}| \leq k_{\max }\right\}$ at $t=0$.) Each of the integral invariants listed in Table 1 fluctuated no more than a few parts per million during their respective simulations.

In each of the simulations, the initial spectra satisfied $|\mathbf{u}(\mathbf{k})|^{2} \sim|\mathbf{b}(\mathbf{k})|^{2} \sim k^{4} \exp \left(-2 k^{2} / k_{o}^{2}\right)$ with $k_{o}=2$ (although the exact shape of the initial spectra was not critical; the critical initial quantities were the values of the total energy and helicities). Time-averages of the components of $\overline{\boldsymbol{\omega}}(\mathbf{k})$ and $\tilde{\mathbf{b}}(\mathbf{k})$ as well as of their squares $\left(\overline{\left|b_{x R}(\mathbf{k})\right|^{2}}, \mathrm{R}\right.$ : real, etc.) were taken, averaging every 50 time steps. (There are six 'components' of $\boldsymbol{\omega}(\mathbf{k})$ and six of $\mathbf{b}(\mathbf{k})$, since the $x-, y$-, and $z$-components of these each have a real and an imaginary part.) The second-order moments which correspond to the same value of $k=|\mathbf{k}|$ can be combined to produce 
average modal energies

$$
E_{k}(k)=\frac{1}{2 n_{k}} \sum_{k} \overline{|\mathbf{u}(\mathbf{k})|^{2}} \quad \text { and } \quad E_{m}(k)=\frac{1}{2 n_{k}} \sum_{k} \overline{|\mathbf{b}(\mathbf{k})|^{2}}
$$

where the sum $\sum_{k}$ is only over all $\mathbf{k}$ with the same value of $k=|\mathbf{k}|$ and where $n_{k}$ is the number of wave vectors $\mathbf{k}$ in such a sum. The modal values of enstrophy and mean squared current were then $\Omega=k^{2} E_{k}(k)$ and $J=k^{2} E_{m}(k)$, respectively.

These modal energies can be compared with those obtained from ensemble-averaging, once the various distribution parameters are found, as described previously. Remember that the distribution parameters for the Euler cases depended on the average value of the enstrophy $\Omega$, while those for the MHD cases depended on the average ratio of magnetic to kinetic energy $R$. For example, in the MHD run M1A, a time-average yields $R_{t}=1.50795$, equilibrium entropy minimization gives $R_{e}=1.50799$, and minimizing the root-meansquare (rms) error between time- and ensemble-average modal energy spectra produces $R_{r m s}=1.50599$. Using the rms values in all cases gives the distribution parameters presented in Table 2.

To see how well the time- and ensemble-averaged modal energy spectra compare, consider Figure 1, where representative spectra are shown ( $\log \equiv \log _{10}$ in the Figures). Figure 1a corresponds to case M2A where $k_{\min }=1$; the goodness of fit is typical of all the Euler and MHD runs. Figure 1b corresponds to case M3A where $k_{\min }=2$; the initial values of the $\boldsymbol{\omega}(\mathbf{k})$ and $\mathbf{b}(\mathbf{k})$ for M3A were the same as those of M2A except that all of the $|\mathbf{k}|=1$ coefficients were set to zero and not allowed to grow during the simulation. These graphs show the average energy for modes with the same $k=|\mathbf{k}|$; since there are twice as many modes with $k=2$ as with $k=1$, Figures $1 \mathrm{a}$ and $1 \mathrm{~b}$ indicate that the modes with $k=k_{\min }$ contain the same total energy. The exact value of $k_{\min }$ is thus not qualitatively important in any given simulation.

It is informative to consider the exact values taken by the Fourier modes during their time evolution. For example, the values of $\boldsymbol{\omega}(\mathbf{k})$ and $\mathbf{b}(\mathbf{k})$ for $\mathbf{k}=(1,0,0)$ for run M1A are shown in Figure 2, where the time evolution is shown by plotting the real vs the imaginary parts of the coefficients for all times between $t=0$ and $t=1000$. The behavior is decidedly non-ergodic since the components clearly do not have zero mean values, and thus do not match the ensemble prediction. It is interesting to note that although average modal behavior (Figure 1) is essentially as predicted, the detailed evolution of the modal coefficients is not. Also, notice in Figure 2 that we appear to have

$$
\omega_{y} \sim i \omega_{z} \quad \text { and } \quad b_{y} \sim i b_{z}
$$


This relationship was also apparent for all the other coefficients which were examined, as long as the coefficient mean values were sufficiently larger than the corresponding standard deviation. The relationship (14) is, in fact, $\mathbf{b} \sim \nabla \times \mathbf{b}$, i.e., $\mathbf{j} \times \mathbf{b} \simeq 0$. In other words, the ideal MHD has become force-free [11], apparently on a mode-by-mode basis.

The average coefficients $\overline{\boldsymbol{\omega}}(\mathbf{k})$ and $\overline{\mathbf{b}}(\mathbf{k})$ can be thought of as the coherent structure underlying these simulations of ideal, isotropic turbulence. These coefficients can be used to calculate the coherent energy present in the turbulence:

$$
E_{k}^{c}(k)=\frac{1}{2 n_{k}} \sum_{k}|\overline{\mathbf{u}}(\mathbf{k})|^{2} \quad \text { and } \quad E_{m}^{c}(k)=\frac{1}{2 n_{k}} \sum_{k}|\overline{\mathbf{b}}(\mathbf{k})|^{2}
$$

(The various helicities can also be calculated in a similar manner.) The average coefficients exist for all cases at $t=250$, so that a comparison can be made; the results of this are presented in Table 3 . Notice that the coherent quantities in Table 3 are a sizeable fraction of the corresponding total quantities in Table 1; for example, the coherent energy in run M1A is $18.8 \%$ of the total energy, while the coherent magnetic helicity in the same run is $83 \%$ of the total!

For those runs which went beyond $t=250$, the coherent energies between $t=520$ and $t=750$ are shown in Figure 3. It is evident that the MHD simulations with $B_{o}=0$ (M1A and M2A) have the largest coherent energies, and that this coherent energy is primarily magnetic. The MHD case with $B_{0}=1$ (M4A) has effectively no coherent energy, while in the Euler case (E2A), the coherent energy is clearly decaying (although still $3 \%$ of the total at $t=750$ ).

\section{Broken Symmetry}

In a Fourier representation (3), the basic equations (1) and (2) take the form

$$
\begin{aligned}
& \dot{\mathbf{b}}(\mathbf{k})=i \mathbf{k} \times \sum_{\mathbf{p}+\mathbf{q}=\mathbf{k}}[\mathbf{u}(\mathbf{p}) \times \mathbf{b}(\mathbf{q})]+i\left(\mathbf{k} \cdot \mathbf{B}_{0}\right) \mathbf{u}(\mathbf{k}) \\
& \dot{\boldsymbol{\omega}}(\mathbf{k})=i \mathbf{k} \times \sum_{\mathbf{p}+\mathbf{q}=\mathbf{k}}[\mathbf{u}(\mathbf{p}) \times \boldsymbol{\omega}(\mathbf{q})+\mathbf{j}(\mathbf{p}) \times \mathbf{b}(\mathbf{q})]+i\left(\mathbf{k} \cdot \mathbf{B}_{o}\right) \mathbf{j}(\mathbf{k})
\end{aligned}
$$

These equations have the symmetry (i.e., invariance under) $\boldsymbol{\omega}(\mathbf{k}) \rightarrow e^{\mathbf{i} \mathbf{k} \cdot \mathbf{a}} \boldsymbol{\omega}(\mathbf{k}), \mathbf{b}(\mathbf{k}) \rightarrow e^{i \mathbf{k} \cdot \mathbf{a}} \mathbf{b}(\mathbf{k})$ which merely reflects the isotropy of space: i.e., place $\mathbf{x} \rightarrow \mathbf{x}+\mathbf{a}$ into (3). However, an average over the displacements a merely reproduces the known fact that the $\mathbf{k}=0$ modes of $\boldsymbol{\omega}$ and $\mathbf{b}$ are zero and remain so, i.e., do 
not participate in the dynamics of the ideal MHD turbulence.

In the case where $\mathbf{B}_{\circ}=0$, the equations (16) again also have the symmetry $\mathbf{b} \rightarrow-\mathbf{b}$; the import of this is that all members of the ensemble of possible realizations of ideal MHD turbulence are, in fact, paired: if $\left\{\mathbf{u}(\mathbf{k}), \mathbf{b}(\mathbf{k}) ; k_{\min } \leq|\mathbf{k}| \leq k_{\max }\right\}$ is a possible realization, then so is $\left\{\mathbf{u}(\mathbf{k}),-\mathbf{b}(\mathbf{k}) ; k_{\min } \leq|\mathbf{k}| \leq k_{\max }\right\}$.

Thus what is calculated in the ensemble average $\langle\mathbf{b}(\mathbf{k})\rangle$ is really $\left\langle\left[\mathbf{b}_{+}(\mathbf{k})+\mathbf{b}_{-}(\mathbf{k})\right] / \mathbf{2}\right\rangle$, where $\mathbf{b}_{+}(\mathbf{k})=$ $\mathbf{b}(\mathbf{k})$ and $\mathbf{b}_{-}(\mathbf{k})=-\mathbf{b}(\mathbf{k})$, in which case $\langle\mathbf{b}(\mathbf{k})\rangle \equiv 0$ automatically. However, $\left\langle\mathbf{b}_{+}(\mathbf{k})\right\rangle$ and $\left\langle\mathbf{b}_{-}(\mathbf{k})\right\rangle$ are undefined; if they are non-zero, then the ensemble symmetry is said to be broken in a given realization (and the dynamics are non-ergodic). That they can be non-zero, and substantially so, has been demonstrated in the numerical results presented herein.

\section{Conclusion}

In this paper, a numerical study of 3D Euler and ideal MHD turbulence was presented. A number of different cases were presented in which the dynamic evolution was allowed to proceed for a considerable length of dimensionless simulation time. These results clearly demonstrate the presence of broken symmetry, non-ergodicity, and coherent structure in ideal 3D MHD turbulence.

Realistic MHD turbulence (except in superfluids) is not ideal since resistive and viscous processes dissipate energy. The results presented here then address, rather than practical cases, only numerical simulations in which fluid and magnetic dissipation can be set identically equal to zero. For these simulations, however, the ideal theory provides a very useful means of testing computer codes.

An interesting, open question is: Does the non-ergodic behavior seen in the ideal magnetohydrodynamic case also occur in real geophysical and astrophysical systems where dynamo activity has been observed [11]? An answer to this question, however, requires the introduction of dissipation into numerical simulations, along with a substantial increase in grid size. Although this was beyond the scope of the present work, it is a straightforward extension of it, provided that sufficiently large computers can be utilized.

Additionally, it should be mentioned that there has been some very interesting work in dynamical systems theory concerning broken symmetry [12]. A full discussion of any connection to the numerical work presented here is also beyond the scope of the present work.

Finally, I would like to thank Dr. M. Y. Hussaini for the opportunity to spend a year in residence at 
ICASE as a Visiting Scientist, and to thank Professor Geoffrey Lilley for his useful comments concerning this paper.

\section{References}

[1] J. V. Shebalin, Physica D, 37, 173-191, 1989.

[2] D. Fyfe \& D. Montgomery, J. Plasmas Phys., 16, 181-191, 1976.

[3] M. Lesieur, Turbulence in Fluids, Chp. X, Kluwer, Dordrecht, 1990.

[4] H. K. Moffatt, J. Fluid Mech., 35, 117-129, 1969.

[5] R. H. Kraichnan, J. Fluid Mech., 59, 745-752, 1973.

[6] U. Frisch, A. Pouquet, J. Leorat, \& A. Mazure, J. Fluid Mech., 68, 769-778, 1975.

[7] A. I. Khinchin, Mathematical Foundations of Statistical Mechanics, Dover, New York, 1949.

[8] J. Gazdag, J. Comp. Phys., 20, 196-207, 1976.

[9] C. Canuto, M. Y. Hussaini, A. Quateroni, \& T.A. Zang, Spectral Methods in Fluid Dynamics, SpringerVerlag, New York, 1988.

[10] G. S. Patterson, Jr., \& S. A. Orszag, Phys. Fluids, 14, 2538-2541, 1971.

[11] H. K. Moffatt, Magnetic Field Generation in Electrically Conducting Fluids, Cambridge Univ. Press, Cambridge, 1971.

[12] M. Golubitsky, I. Steward, \& D. G. Schaeffer, Singularities and Groups in Bifurcation Theory, SpringerVerlag, New York, 1988. 
Table 1. Numerical Simulations

\begin{tabular}{|c|c|c|c|c|c|c|c|c|}
\hline \multirow{2}{*}{ Run } & $\frac{c p u-s e c}{\Delta t}$ & \multirow{2}{*}{$k_{m i n}$} & \multirow{2}{*}{$B_{o}$} & Total & \multicolumn{5}{|c|}{ Integral Invariants } \\
\cline { 5 - 9 } & & & & Time & $E$ & $H_{k}$ & $H_{c}$ & $H_{m}$ \\
\hline E1A & 0.12 & 1 & - & 250 & 0.5000 & -.04443 & - & - \\
\hline E2A & 0.12 & 1 & - & 750 & 0.5000 & 3.142 & - & - \\
\hline E2R & 0.25 & 1 & - & 250 & 0.5000 & 3.142 & - & - \\
\hline \hline M1A & 0.35 & 1 & 0 & 1000 & 1.000 & - & 0.1326 & 0.2129 \\
\hline M1R & 0.71 & 1 & 0 & 250 & 1.000 & - & 0.1326 & 0.2129 \\
\hline M2A & 0.35 & 1 & 0 & 750 & 1.000 & - & 0.1326 & 0.09973 \\
\hline M3A & 0.35 & 2 & 0 & 250 & 0.9983 & - & 0.1258 & 0.09899 \\
\hline M4A & 0.35 & 1 & 1 & 750 & 1.000 & - & 0.1326 & - \\
\hline
\end{tabular}

Table 2. Distribution Parameters

\begin{tabular}{|c|c|c|c|c|c|}
\hline Run & $\alpha$ & $\beta$ & $\gamma$ & $\Omega_{r m s}$ & $R_{r m s}$ \\
\hline \hline E1A & 2.624 & 0.006825 & - & 17.08 & - \\
\hline E2A & 15.58 & -2.062 & - & 23.74 & - \\
\hline E2R & 15.41 & -2.035 & - & 23.79 & - \\
\hline \hline M1A & 1.774 & -0.7825 & -1.682 & - & 1.506 \\
\hline M1R & 1.773 & -0.7824 & -1.681 & - & 1.506 \\
\hline M2A & 1.547 & -0.7142 & -1.451 & - & 1.206 \\
\hline M3A & 1.616 & -0.7205 & -2.131 & - & 1.301 \\
\hline M4A & 1.401 & -0.7075 & - & - & 1.000 \\
\hline
\end{tabular}


Table 3. Coherency at $t=250$

\begin{tabular}{|l|l|l|l|l|l|}
\hline Run & $E_{k}^{c}$ & $E_{m}^{c}$ & $H_{k}^{c}$ & $H_{c}^{c}$ & $H_{m}^{c}$ \\
\hline \hline E1A & 0.00301 & - & -.00110 & - & - \\
\hline E2A & 0.0416 & - & 0.305 & - & - \\
\hline E2R & 0.0413 & - & 0.303 & - & - \\
\hline \hline M1A & 0.0880 & 0.179 & - & 0.0364 & 0.177 \\
\hline M1R & 0.0919 & 0.172 & - & 0.0365 & 0.170 \\
\hline M2A & 0.00562 & 0.0656 & - & 0.0158 & 0.0635 \\
\hline M3A & 0.00464 & 0.0542 & - & 0.0126 & 0.0368 \\
\hline M4A & 0.00146 & 0.00148 & - & 0.000421 & 0.0000955 \\
\hline
\end{tabular}



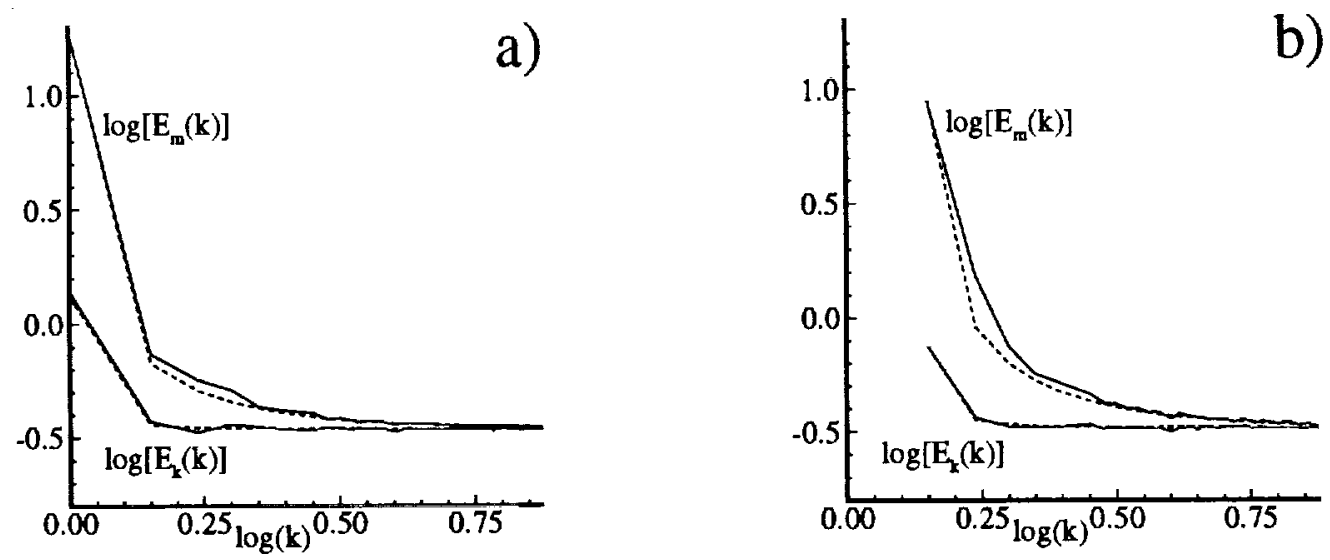

Figure 1. Energy spectra for a) M2A and b) M3A; - - - theoretical, ------- numerical.
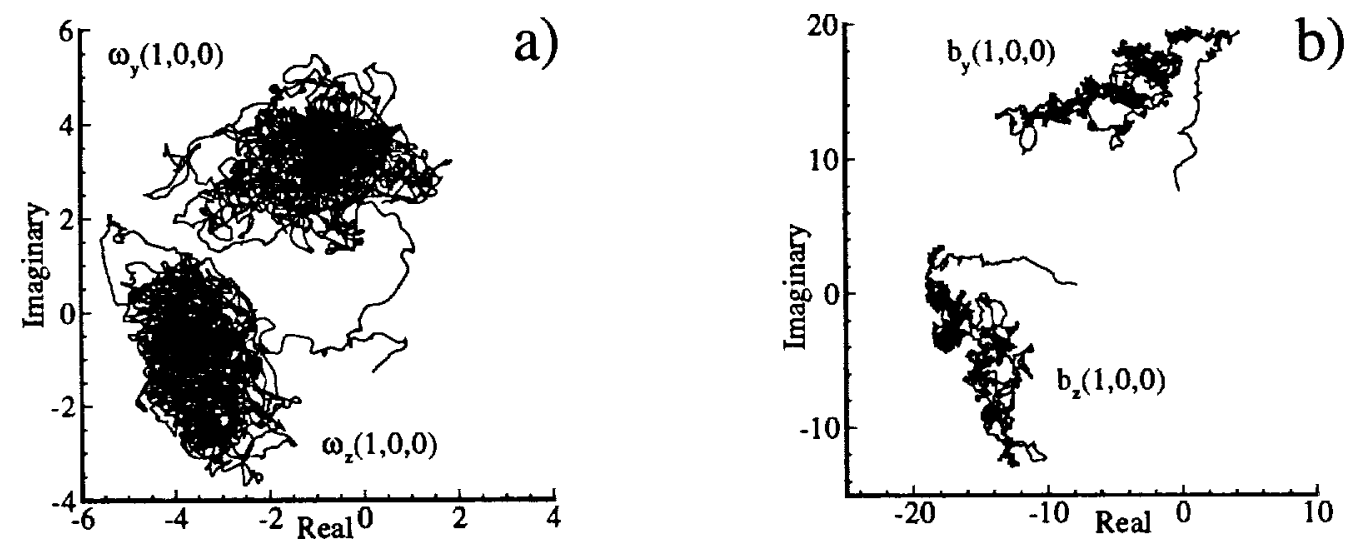

Figure 2. Components of two vector modes of M1A as they evolve from $t=0$ to 1000 : a) vorticity and b) magnetic field.

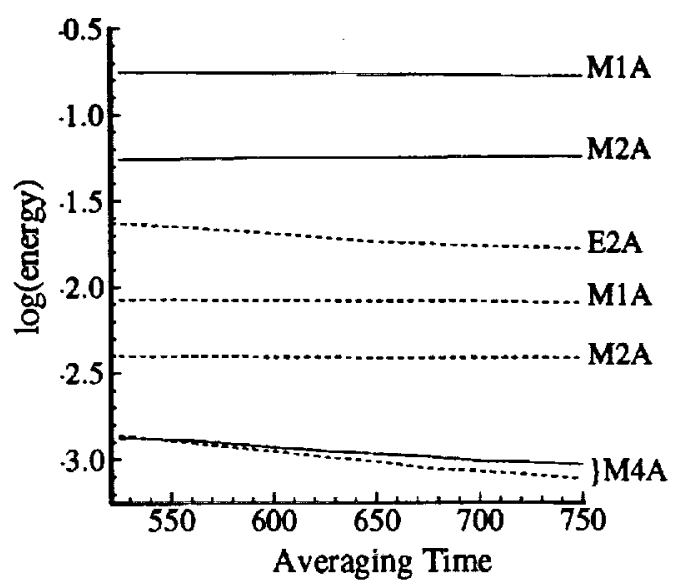

Figure 3. Coherent energies: - - - kinetic, ------- magnetic. 



\begin{tabular}{|c|c|c|c|c|}
\hline \multicolumn{3}{|c|}{ REPORT DOCUMENTATION PAGE } & \multicolumn{2}{|r|}{$\begin{array}{l}\text { Form Approved } \\
\text { OMB No. 0704-0188 }\end{array}$} \\
\hline \multicolumn{5}{|c|}{ 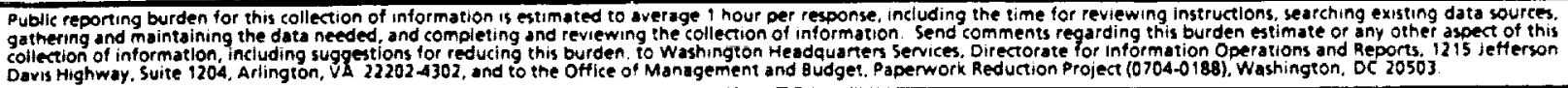 } \\
\hline \multicolumn{2}{|c|}{\begin{tabular}{l|l} 
1. AGENCY USE ONLY (Leave blank) & $\begin{array}{l}\text { 2. REPORT DATE } \\
\text { August } 1993\end{array}$
\end{tabular}} & \multicolumn{3}{|c|}{$\begin{array}{l}\text { 3. REPORT TYPE AND DATES COVERED } \\
\text { Technical Memorandum }\end{array}$} \\
\hline \multicolumn{3}{|c|}{$\begin{array}{l}\text { 4. TITLE AND SUBTITLE } \\
\text { BROKEN SYMMETRY IN IDEAL MAGNETOHYDRODYNAMIC TURBULENCE }\end{array}$} & \multirow{2}{*}{\multicolumn{2}{|c|}{$\begin{array}{l}\text { 5. FUNDING NUMBERS } \\
\text { WU 505-90-52-01 }\end{array}$}} \\
\hline \multicolumn{3}{|l|}{$\begin{array}{l}\text { 6. AUTHOR(S) } \\
\text { John V. Sheballn }\end{array}$} & & \\
\hline \multicolumn{3}{|c|}{$\begin{array}{l}\text { 7. PERFORMING ORGAMIZATION NAME(S) AND ADDRESS(ES) } \\
\text { Inst I tute for Computer Applications in Science } \\
\text { and Engineering } \\
\text { Ma11 Stop 132C, NASA Langley Research Center } \\
\text { Hampton, VA 23681-0001 }\end{array}$} & \multicolumn{2}{|c|}{$\begin{array}{l}\text { 8. PERFORMING ORGANIZATION } \\
\text { REPORT NUMBER } \\
\text { ICASE RepOrt No. } 93-49\end{array}$} \\
\hline \multicolumn{3}{|c|}{$\begin{array}{l}\text { 9. SPONSORING/MONITORING AGENCY NAME(S) AND ADDRESS(ES) } \\
\text { National Aeronautics and Space Adminlstration } \\
\text { Langley Research Center } \\
\text { Hampton, VA 23681-0001 }\end{array}$} & \multicolumn{2}{|c|}{$\begin{array}{l}\text { 10. SPONSORING/MONITORING } \\
\text { AGENCY REPORT NUMBER } \\
\text { NASA TM-109017 } \\
\text { ICASE RePOIT NO. } 93-49\end{array}$} \\
\hline \multicolumn{5}{|c|}{$\begin{array}{l}\text { T1. SUPPLEMENTARY NOTES } \\
\text { Langley Technical Monitor: Michael F. Card Submitted to Physics of Fluids B } \\
\text { Final Report }\end{array}$} \\
\hline \multicolumn{3}{|c|}{$\begin{array}{l}\text { 12a. DISTRIBUTION/AVALABBLITY STATEMENT } \\
\text { Unclassified - Unlimited } \\
\text { Subject Category } 34\end{array}$} & \multicolumn{2}{|c|}{ 12b. DISTRIBUTION CODE } \\
\hline \multicolumn{5}{|c|}{ 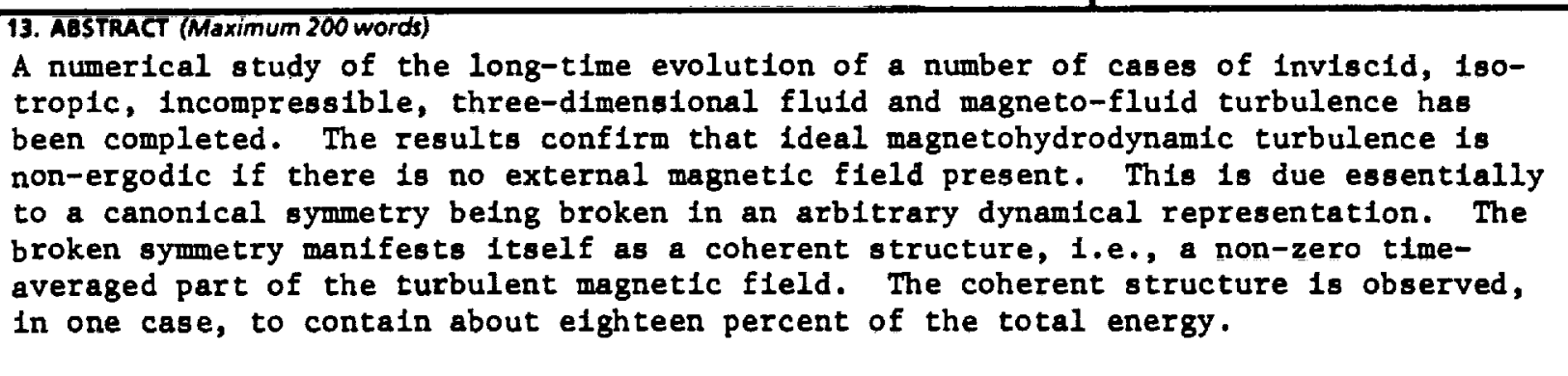 } \\
\hline \multicolumn{4}{|c|}{$\begin{array}{l}\text { 14. SUBJEC TERMS } \\
\text { 1sotropic turbulence, 1deal magnetohydrodynamics, broken } \\
\text { symmetry }\end{array}$} & $\begin{array}{l}\text { 15. NUMBER OF PAGES } \\
17 \\
\begin{array}{l}\text { 16. PRICE CODE } \\
\mathrm{A0} 3\end{array}\end{array}$ \\
\hline $\begin{array}{l}\text { 17. SECURITY CLASSIFICATION } \\
\text { OF REPORT } \\
\text { Unclassifled }\end{array}$ & $\begin{array}{l}\text { 18. SECURITY CLASSIFICATION } \\
\text { OF THIS PAGE } \\
\text {.UnClassified }\end{array}$ & 19. SE & CATION & 20. LIMITATION OF ABSTRACT \\
\hline
\end{tabular}

